\title{
Avaliação diagnóstica da Medicina Tradicional Chinesa dos sintomas de estresse tratados pela auriculoterapia: ensaio clínico
}

Traditional Chinese Medicine diagnostic evaluation of stress symptoms treated by auriculotherapy: a clinical trial Evaluación diagnóstica de Medicina Tradicional China de los síntomas de estrés tratados

por auriculoterapia: ensayo clínico

Leonice Fumiko Sato Kurebayashi ${ }^{1}$, Juliana Rizzo Gnatta ${ }^{2}$, Talita Pavarini Borges ${ }^{3}$, Maria Júlia Paes da Silva ${ }^{4}$ ${ }^{1}$ Enfermeira acupunturista, Mestre em Enfermagem. Discente do Programa de Pós-Graduação em Enfermagem na Saúde do Adulto e do Idoso (PROESA), nível Doutorado, da Escola de Enfermagem da Universidade de São Paulo (EE/USP). São Paulo, São Paulo, Brasil. E-mail: fumie ibez@yahoo.com.br. 2 Enfermeira, Mestre em Ciências da Saúde. Discente do PROESA/EE/USP, nível Doutorado. São Paulo, São Paulo, Brasil. E-mail: juliana.gnatta@usp.br.

3 Enfermeira. Discente do PROESA/EE/USP, nível Mestrado. São Paulo, São Paulo, Brasil. E-mail: talita pavarini@yahoo.com.br.

${ }^{4}$ Enfermeira, Doutora em Enfermagem. Professora Titular da EE/USP. São Paulo, São Paulo, Brasil. E-mail: juliaps@usp.br.

\section{RESUMO}

Ensaio clínico que objetivou identificar diagnósticos da Medicina Tradicional Chinesa (MTC) a partir de sintomas de estresse que foram responsivos ao tratamento por auriculoterapia. Setenta e cinco profissionais da equipe de Enfermagem de um Hospital-Escola com médio e alto escore de estresse pela Lista de Sintomas de Estresse de Vasconcelos foram randomizados em 3 grupos (Controle sem tratamento, Auriculoterapia com agulhas e Auriculoterapia com sementes), tratados semanalmente nos pontos Rim, Tronco cerebral e Shenmen, por 8 sessões. A Análise de Variância demonstrou diferenças significativas após o tratamento $(p=0.023)$ somente entre os grupos agulha e controle, sendo que 21 sintomas apresentaram diferenças $(p<0.05)$ pelo teste pareado $t$-Student. Concluiu-se que a auriculoterapia reduziu o estresse com melhores resultados para agulhas do que para sementes e os principais diagnósticos encontrados foram: Estagnação de Qi no tórax, Estagnação do Fígado e dos meridianos tendino-musculares, Deficiência de Yin do Fígado, Rins e Xue (Sangue).

Descritores: Enfermagem; Auriculoterapia; Medicina Tradicional Chinesa; Diagnóstico.

\section{ABSTRACT}

The objective of this clinical trial was to identify the Traditional Chinese Medicine (TCM) diagnoses for stress symptoms that responded to auriculotherapy. Subjects were 75 nursing professionals of a teaching hospital, with average and high stress scores according to Vasconcelos' List of Stress Symptoms. The subjects were randomized into three groups (control without treatment, auriculotherapy with needles, and auriculotherapy with seeds), treated weekly in the Kidney, Brain stem and Shenmen, for eight sessions. Analysis of variance showed significant differences after treatment $(p=0.023)$ only between the needle and group controls, with 21 symptoms showing differences $(p<0.05)$ in the Student's t-test. In conclusion, auriculotherapy reduced stress with best result for needles than seeds, and the main identified diagnoses were: Stagnation of Qi in the chest, Stagnation of the Liver and the tendinomuscular meridians, Yin Deficiency of the Liver, Kidneys and Xue (Blood).

Descriptors: Nursing; Auriculotherapy; Medicine, Chinese Traditional; Diagnosis.

\section{RESUMEN}

Ensayo clínico objetivando identificar diagnósticos de la Medicina Tradicional China (MTC) a partir de síntomas de estrés responsivos al tratamiento de auriculoterapia. Setenta y cinco profesionales del Equipo de Enfermería de un HospitalEscuela con puntajes medios y altos de estrés según Lista de Síntomas de Estrés de Vasconcelos, fueron divididos en tres grupos aleatorios (Control sin tratamiento, Auriculoterapia con agujas y Auriculoterapia con semillas), tratados semanalmente en los puntos Riñón, Tronco Cerebral y Shenmen, durante 8 sesiones. El Análisis de Varianza demostró diferencias significativas luego del tratamiento $(p=0.023)$ sólo entre los grupos "aguja" y "control", habiendo expresado diferencias $(p<0.05) 21$ síntomas por el test pareado $t$-Student. Se concluyó en que la auriculoterapia redujo el estrés, con mejores resultados para agujas que para semillas. Los diagnósticos más encontrados fueron: Estancamiento de Qi en el tórax, Estancamiento del Hígado y los meridianos tendinoso-musculares, Deficiencia de Yin del Hígado, Riñones y Xue (Sangre).

Descriptores: Enfermería; Auriculoterapia; Medicina China Tradicional; Diagnóstico. 


\section{INTRODUÇÃO}

O impacto negativo do estresse laboral sobre a qualidade de vida dos trabalhadores tem sido foco de atenção, e estudos têm sido realizados sobre o estresse na equipe de Enfermagem, identificando as principais fontes geradoras de estresse na atuação do enfermeiro, nas diferentes áreas de instituição hospitalar ${ }^{(1-2)}$. A equipe de Enfermagem constitui a maior força de trabalho no contexto hospitalar e as atividades realizadas pelos seus profissionais são marcadas por diversos fatores condicionantes negativos, entre eles, a divisão fragmentada de tarefas, rígida estrutura hierárquica para o cumprimento de rotinas, normas e regulamentos, dimensionamento qualitativo e quantitativo insuficiente de pessoal ${ }^{(3)}$.

Em estudo transversal realizado em Pronto-Socorro encontrou-se que a maioria das trabalhadoras de Enfermagem apresentavam sintomas de estresse de resistência e estágios de quase-exaustão, com a presença de sintomas físicos e psicológicos ${ }^{(4)}$ e tem se discutido a urgência de se investir na qualidade de vida no trabalho dos profissionais de Enfermagem ${ }^{(5)}$, pois altos níveis de estresse podem ter efeitos debilitantes que reduzem a capacidade de trabalhar, aumentando os índices de absenteísmo, afastamento por doenças, comprometendo a habilidade de lidar com situações desafiadoras ${ }^{(6)}$.

Neste sentido, a auriculoterapia, uma das modalidades da Medicina Tradicional Chinesa (MTC), é uma intervenção que poderia ser incluída no âmbito das tecnologias preventivas e de redução de riscos para o adoecimento, em função do estresse de alto nível e de ocorrência prolongada entre profissionais de Enfermagem. Esta modalidade foi avaliada como uma alternativa que melhorou o coping da equipe de Enfermagem ${ }^{(7)}$ e reduziu os níveis de estresse entre profissionais de Enfermagem em Unidade de Terapia Intensiva( ${ }^{(8)}$.

Para a MTC, uma das principais etiologias para o adoecimento e que são consideradas de origem interna são os aspectos psicológicos e as emoções. Condições estressantes podem levar a uma má distribuição energética nos meridianos (canais por onde circula o Qi ou energia), órgãos e vísceras, desencadeando doenças físicas, mentais e emocionais. A MTC busca entender e tratar o indivíduo em sua complexidade e como um todo, estabelecendo diagnósticos energéticos prévios e utilizando um conjunto de técnicas orientais: acupuntura, moxabustão, acupressura, fitoterapia, exercícios físicos, auriculoterapia, reflexologia dos pés, craniopuntura entre outros $^{(9)}$.
Na prática clínica diária, para a realização da avalição diagnóstica de MTC, o padrão de desequilíbrio é identificado a partir de quatro critérios: inspeção, olfação, palpação e interrogatório, sendo possível concluir sobre as condições patológicas em termos de síndromes ou Zheng em chinês. São estes padrões energéticos desequilibrados (Zheng) entendidos na individualidade de cada um que permitem a adequação do tratamento para que menores efeitos colaterais surjam. O diagnóstico é definido para cada pessoa e a cada momento do tratamento(10). Por outro lado, o uso de protocolo é uma forte recomendação das Práticas Baseadas em Evidências ${ }^{(11)}$ e o presente ensaio optou por fazer uso de um protocolo de três pontos auriculares para estresse com base na literatura.

$\mathrm{Na}$ auriculoterapia, a ideia central é que toda parte do corpo tem um ponto reflexo correspondente na orelha, que pode ser utilizado para diagnóstico e tratamento. $O$ efeito positivo da auriculoterapia para redução dos níveis de estresse, está relacionado à diminuição da liberação do hormônio cortisol, que é um importante marcador dos níveis de estresse e tem um forte efeito antiinflamatório(12).

Embora pesquisas sobre estresse estejam sendo realizadas no campo da acupuntura e da auriculoterapia nas últimas décadas(13-14), há carência de artigos científicos que busquem contribuir para o entendimento da linguagem específica e particular da MTC quanto aos padrões de desequilíbrio energético envolvidos no estresse, formas de diagnóstico e tratamento. Uma das formas de avaliação da eficácia da terapêutica segundo a MTC é fundamentada na composição das sensações de bem-estar do paciente com a análise da variabilidade dos sinais clínicos previamente encontrados ${ }^{(15)}$.

Tendo em vista tais informações, o presente estudo teve como objetivo identificar os principais diagnósticos de MTC, a partir da análise dos sintomas da Lista de Sintomas de Stress ${ }^{(16)}$ que melhor responderam ao tratamento de auriculoterapia.

\section{METODOLOGIA}

Trata-se de um Ensaio Clínico Randomizado realizado em diferentes setores de um Hospital-Escola em São Paulo (Pronto Socorro Adulto e Infantil, Unidade de Terapia Intensiva Adulto e Pediátrica, Clínica Médica, Clínica Cirúrgica, Central de Material e Esterilização e Central de Controle de Infecção Hospitalar, Pediatria, Berçário e Alojamento Conjunto).

Da população de 850 profissionais de Enfermagem do hospital, 109 deles, entre enfermeiros, técnicos e auxiliares, compuseram a amostra e 75 conseguiram 
finalizar a pesquisa. Para definir a amostra foi utilizada a Lista de Sintomas de Stress (LSS) de Vasconcelos, que foi aplicada para aqueles que manifestaram interesse em participar. Entretanto, apenas os indivíduos que atenderam aos critérios de inclusão foram convidados. Os participantes foram distribuídos em três grupos, "Grupo Controle" (sem tratamento), "Grupo Auriculoterapia com Agulhas Semipermanentes" e "Grupo Auriculoterapia com Sementes". A alocação dos participantes foi feita por meio de envelopes opacos e numerados e posteriormente sorteados em programa de computador com números gerados no site http://www.randomizer.org/. Dos 75 profissionais de Enfermagem que finalizaram, 22 sujeitos eram do Grupo Controle, 27 do Grupo Auriculoterapia com agulhas semipermanentes e 26 do Grupo Sementes.
Os critérios de inclusão foram: participação voluntária no estudo com disponibilidade de horário para submissão as oito sessões; obtenção dos escores mínimos do LSS para médio e alto. Os critérios de exclusão foram: gravidez, licença médica ou férias durante a pesquisa; qualquer perda consecutiva de sessão; efeitos colaterais, questionários incompletos, entre outros (Figura 1). Não foram excluídos aqueles com enfermidades autoreferidas, pois os pontos do protocolo são pontos gerais para redução de estresse e que podem ser utilizados sem restrições. A única exceção seria a litíase renal, pois o ponto auricular do rim pode estimular a eliminação de pedras renais. Tal efeito pode ser utilizado intencionalmente, mas se sugere o acompanhamento médico.

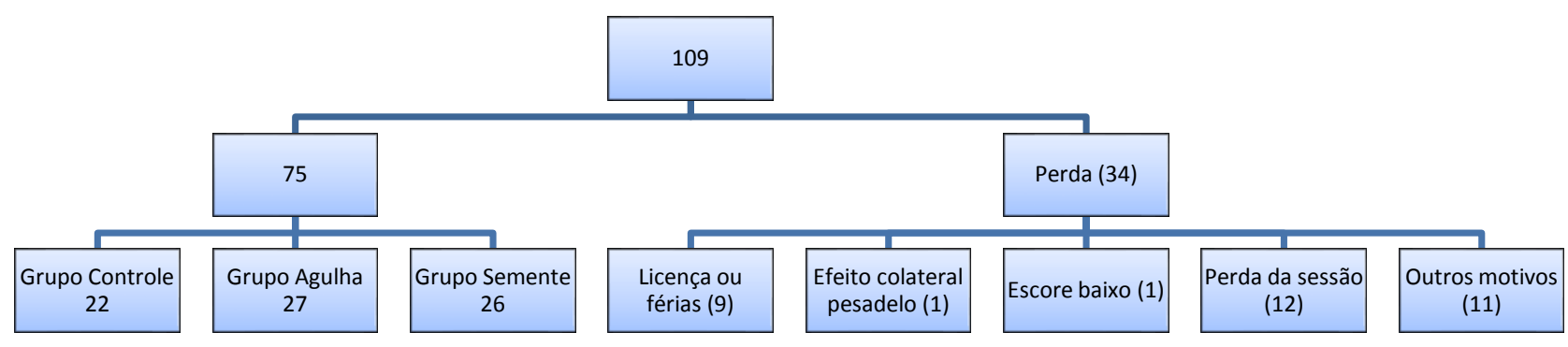

Figura 1: Diagrama de flutuação dos sujeitos da pesquisa. São Paulo, São Paulo, Brasil, 2010.

A coleta de dados foi realizada no primeiro semestre de 2010 em um Hospital em São Paulo. Foi aplicada a Lista de Sintomas de Estresse. Na LSS, com 59 itens, deve-se assinalar a frequência com que ocorrem os sintomas, a partir de uma escala de (0) nunca, (1) raramente, (2) frequentemente e (3) sempre. A pontuação total desse inventário pode variar de 0 a 177 pontos, sendo que os escores mais baixos indicam ausência de estresse ( 0 a 11 pontos), baixo nível (12 a 28), médio nível (29 a 60), alto nível (61 a 120) e altíssimo nível (acima de 120 pontos).

Os Grupos de Auriculoterapia com agulhas e sementes receberam oito sessões (uma por semana, nos pontos Shenmen e Tronco Cerebral. Os pontos Shenmen e Tronco Cerebral têm propriedades calmantes ${ }^{(17)}$. Nos atendimentos protocolares com pontos previamente definidos não se faz uma avaliação diagnóstica a cada sessão, o que justifica a rapidez da colocação dos três pontos auriculares. As sessões duraram, em média, de cinco a dez minutos. Para a colocação das agulhas semipermanentes, após a devida localização dos pontos reativos com um localizador de pontos, foi utilizada a antissepsia com algodão e álcool etílico $70 \%$ do pavilhão auricular e aplicação de agulhas estéreis afixadas com fita adesiva. Quanto às medidas de segurança, recomendações para a acupuntura podem ser comparadas àquelas para a inserção de cateteres intravasculares descritas pelos Centros para Controle e Prevenção de Doenças dos Estados Unidos (United States Centers for Disease Control and Prevention - CDC), ou seja, a higiene simples das mãos, com água e sabão, ou álcool gel desinfetante antes de realizar o procedimento, técnica asséptica garantindo a esterilidade do material e antissepsia da pele com solução de álcool(18). Foram realizadas oito sessões, pois este foi o número de atendimentos a partir do qual resultados positivos de redução de estresse foram alcançados em estudo realizado para a equipe de Enfermagem de Unidade de Terapia Intensiva de um Hospital particular em São Paulo(8).

Para a descrição e análise dos dados foram consideradas as medidas de tendência central e de variabilidade, respectivamente, média e desvio padrão. Para a comparação entre as pontuações do LSS nos diferentes momentos da avaliação - LSS1 ou Baseline, LSS2 (na quarta sessão), LSS3 (na oitava sessão) e LSS4 (Follow-up de 15 dias) - foi utilizado o teste de ANOVA para medidas repetidas e o teste de múltiplas comparações de Bonferroni pelo programa SPSS versão 19.0. Para a análise dos principais sintomas que responderam positivamente ao tratamento foram 
escolhidos os dados referentes à primeira e terceira avaliação, isto é, após oito sessões de auriculoterapia com agulhas e sementes. Como houve homocedasticidade e normalidade dos dados, para a análise estatística foi utilizado um teste paramétrico $t$-Student pareado para avaliar o antes e após o tratamento.

O projeto de pesquisa foi aprovado pelo Comitê de Ética em Pesquisa do Hospital Universitário da Universidade de São Paulo (Parecer CEP-HU/USP 941/09) e possui o número de identificação do registro de Ensaios Clínicos no ClinicalTrials.gov (NCT01141374). O estudo atendeu à Resolução 196/1996 do Conselho Nacional de Saúde Brasileiro ao envolver seres humanos na pesquisa, tendo sido explicitado o convite e os objetivos da pesquisa antes da entrega do Termo de Consentimento Livre e Esclarecido aos sujeitos da pesquisa.

\section{RESULTADOS}

Participaram do estudo 75 pessoas que apresentaram escores médio $(44 / 58.7 \%)$ e alto $(31 / 41.3 \%)$ pela Lista de Sintomas de Stress. Na análise de variância (ANOVA) de medidas repetidas, constataram-se diferenças entre os três grupos (controle sem intervenção, auriculoterapia com agulhas, auriculoterapia com sementes) na terceira avaliação $(p=0.023)$ e follow-up $(p=0.003)$. Não foi observada diferença estatisticamente significativa para o grupo sementes quando comparado aos demais, porém, ao analisar os resultados dentro do mesmo grupo, observaram-se diferenças estatísticas $(p<0.05)$, já a partir da segunda avaliação. É importante frisar que os resultados analisados dos sintomas mais responsivos para a identificação dos diagnósticos de MTC foram aqueles obtidos após oito sessões de tratamento, tendo sido excluídos os dados do grupo controle sem intervenção.

Entre os três grupos houve homogeneidade quanto à idade, tempo de trabalho e LSS inicial. Quanto ao sexo houve predominância do sexo feminino (71), com distribuição homogênea dos poucos homens que fizeram parte da pesquisa( ${ }^{(4)}$.

Tabela 1: Média e desvio padrão da idade, tempo de trabalho e escore inicial do LSS e proporção de gênero na amostra. São Paulo, São Paulo, Brasil, 2010.

\begin{tabular}{ccccc}
\hline & Controle & Agulha & Semente & p \\
\hline Idade (anos) & $39.25(13.17)$ & $41.37(8.23)$ & $45.57(6.40)$ & 0.51 \\
Tempo de trabalho (anos) & $13.91(10.70)$ & $14.03(9.34)$ & $15.37(4.07)$ & 0.29 \\
LSS inicial (pontos) & $54.36(15.90)$ & $66.82(18.56)$ & $63.27(26.06)$ & 0.34 \\
Sexo (\%F) & 91.66 & 96.29 & 96.15 & 0.65 \\
\hline
\end{tabular}

Para a análise dos sintomas de estresse mais responsivos ao tratamento, foi extraído dos 75 sujeitos, 22 do grupo controle sem intervenção. Restaram, portanto, 53 pessoas, dos grupos de intervenção. Foram 37 Técnicos de Enfermagem, nove Auxiliares de Enfermagem e sete Enfermeiras, de diversos setores do Hospital: Unidade Básica, UTI-Pediátrica, Centro Cirúrgico, Berçário, Pediatria, Clínica Médica, Clínica Cirúrgica, UTI-Adulto, Pronto Socorro Infantil, Pronto Socorro Adulto, Controle de Materiais e Esterilização, Alojamento conjunto, Centro Obstétrico, Comissão de Controle e Infecção Hospitalar.

Segundo o Teste $t$-Student Pareado, dos 59 itens avaliados, os sintomas que apresentaram diferenças estatísticas após oito sessões de auriculoterapia com agulhas estão enumerados na Tabela 2. 
Tabela 2: Média da diferença, desvio padrão, significância obtida pelo test-t pareado de estresse do Grupo Agulha antes e após oito sessões. São Paulo, São Paulo, Brasil, 2010.

\begin{tabular}{|c|c|c|c|}
\hline Sintomas de Estresse & Média & DP & $\mathbf{p}$ \\
\hline Sinto a respiração ofegante & 0.333 & 0.784 & 0.036 \\
\hline Tenho taquicardia & 0.593 & 0.971 & 0.004 \\
\hline Tenho a sensação que vou desmaiar & 0.259 & 0.594 & 0.032 \\
\hline Tenho desânimo & 0.333 & 0.832 & 0.047 \\
\hline Noto que minhas forças estão no fim & 0.333 & 0.679 & 0.017 \\
\hline Minha pressão arterial se altera & 0.519 & 0.7 & 0.001 \\
\hline Apresento distúrbios gastrintestinais & 0.407 & 0.888 & 0.025 \\
\hline Tenho cansaço & 0.37 & 0.742 & 0.015 \\
\hline Sinto dores nas costas & 0.519 & 0.753 & 0.001 \\
\hline Tenho insônia & 0.259 & 0.526 & 0.017 \\
\hline Sinto raiva & 0.296 & 0.724 & 0.043 \\
\hline Sinto náuseas & 0.259 & 0.526 & 0.017 \\
\hline Sinto os olhos lacrimejantes e a visão embaçada & 0.444 & 1.013 & 0.031 \\
\hline Sinto exaustão física & 0.37 & 0.792 & 0.022 \\
\hline Sinto insegurança & 0.407 & 0.694 & 0.005 \\
\hline Tenho pesadelos & 0.444 & 0.751 & 0.005 \\
\hline Tenho um nó no estômago & 0.481 & 1.051 & 0.025 \\
\hline Sofro de enxaqueca & 0.259 & 0.656 & 0.05 \\
\hline Meu apetite oscila muito & 0.704 & 1.068 & 0.002 \\
\hline Minha vida sexual está difícil & 0.259 & 0.594 & 0.032 \\
\hline Meus músculos estão sempre tensos & 0.481 & 0.975 & 0.016 \\
\hline
\end{tabular}

Com relação ao Grupo Semente, foram encontrados os seguintes sintomas da Lista de Sintomas de Stress que apresentaram diferenças estatisticamente significativas segundo o Teste t-Student pareado (Tabela 3).

Tabela 3: Média da diferença, Desvio Padrão e Significância dos sintomas de estresse antes e após auriculoterapia com sementes. São Paulo, São Paulo, Brasil, 2010.

\begin{tabular}{|c|c|c|c|}
\hline & Média & DP & $\mathbf{p}$ \\
\hline Tenho sensação de que vou desmaiar & 0.462 & 0.905 & 0.015 \\
\hline Tenho desânimo & 0.423 & 0.902 & 0.025 \\
\hline Apresento distúrbios gastrintestinais & 0.385 & 0.898 & 0.039 \\
\hline Tenho insônia & 0.346 & 0.846 & 0.047 \\
\hline Sinto os olhos lacrimejantes e a visão embaçada & 0.5 & 0.949 & 0.013 \\
\hline Tenho dor de cabeça & 0.308 & 0.679 & 0.029 \\
\hline Sofro de enxaqueca & 0.269 & 0.667 & 0.05 \\
\hline Meus músculos estão sempre tensos & 0.462 & 0.761 & 0.005 \\
\hline
\end{tabular}

$\mathrm{Na}$ Tabela 4, serão apresentados os diagnósticos de MTC em análise comparativa com os principais sintomas do LSS que responderam positivamente ao tratamento de auriculoterapia. Cabe ressaltar, porém, que tais diagnósticos são sugestivos e não se pretende esgotar o assunto. Como já explicitado anteriormente, definir diagnósticos depende de um processo complexo em que observação de língua e pulso são fundamentais para o acerto da síndrome (Zheng). 
Tabela 4: Descritiva de Diagnósticos de MTC a partir dos sintomas de estresse mais responsivos após oito sessões de auriculoterapia. São Paulo, São Paulo, Brasil, 2010.

\begin{tabular}{|c|c|}
\hline Sintomas de Estresse & Diagnósticos de MTC \\
\hline Sinto a respiração ofegante & Estagnação de Qi no tórax \\
\hline Tenho taquicardia & Estagnação de Qi no tórax \\
\hline Tenho a sensação que vou desmaiar & Deficiência ou Estagnação de Qi/Xue \\
\hline Tenho desânimo & Deficiência energética geral e do R \\
\hline Noto que minhas forças estão no fim & Deficiência energética geral e do $\mathrm{R}$ \\
\hline Minha pressão arterial se altera & Estagnação de Qi no tórax \\
\hline Apresento distúrbios gastrintestinais & Desarmonia do E e BP \\
\hline Tenho cansaço & Deficiência ou Estagnação de Qi Geral \\
\hline Sinto dores nas costas & Estagnação de Qi/Xue nos tendino-musculares \\
\hline Tenho insônia & Desarmonia do $\mathrm{F}$ e C \\
\hline Sinto raiva & Estagnação de Qi do F \\
\hline Sinto náuseas & Desarmonia do E e BP \\
\hline Olhos lacrimejantes e a visão embaçada & Deficiência de Yin do F \\
\hline Sinto exaustão física & Deficiência energética geral do R \\
\hline Sinto insegurança & Deficiência energética do Re C \\
\hline Tenho pesadelos & Desarmonia do F e C \\
\hline Tenho um nó no estômago & Desarmonia do E e BP \\
\hline Sofro de enxaqueca & Subida de Yang/ Deficiência de Yin do F \\
\hline Meu apetite oscila muito & Desarmonia do E e BP \\
\hline Minha vida sexual está difícil & Deficiência geral de Qi e dos R/Distúrbio do Shen \\
\hline Meus músculos estão sempre tensos & Estagnação de Qi do F \\
\hline
\end{tabular}

Quanto aos efeitos adversos foram poucos os relatados, dentre eles, pesadelo e aumento de ansiedade por comida. Apenas uma participante desistiu com queixas de pesadelos. Esta paciente era do turno noturno e estava sempre cansada e a paciente que sentiu ansiedade por comida, tinha sofrido uma cirurgia bariátrica e perdido muito peso. A auriculoterapia e acupuntura tendem a reequilibrar a distribuição de Qi nos meridianos, órgãos e vísceras ${ }^{(19)}$ e se houver extremo cansaço e deficiência de energia, é possível que ocorram efeitos inesperados. Alguns pacientes relataram também desconforto com a colocação e permanência das agulhas, o que mostra uma desvantagem deste material com relação às sementes. Uma paciente referiu prurido na colocação das sementes e foi escolhido outro material adesivo.

\section{DISCUSSÃO}

A auriculoterapia tem sido utilizada para o tratamento de muitas condições de desequilíbrio energético e também para controle de sintomas como ansiedade e estresse. $O$ resultado positivo deste estudo vem de encontro com outras pesquisas realizadas com auriculoterapia para estresse: auriculoterapia para redução do estresse em Equipe de Enfermagem em Terapia Intensiva (auriculoterapia com agulhas) $^{(8)}$ e outro estudo para ansiedade: eficácia da auriculoterapia no tratamento de ansiedade em 71 estudantes de Enfermagem, um ensaio clínico simples-cego, com grupo controle, auricular e placebo com a utilização dos pontos Shenmen e Tronco
Cerebral(20). No presente estudo, a este protocolo de pontos auriculares foi acrescido o ponto Rim.

Quanto aos sintomas que melhor responderam ao tratamento, observaram-se 21 sintomas no grupo tratado com agulhas semipermanentes e oito sintomas no grupo com sementes, com melhores resultados para agulhas do que para sementes. No estudo de auriculoterapia com agulhas semipermanentes para estresse em 41 colaboradores da equipe de Enfermagem de CTI, os principais sintomas apresentados foram: desgaste, dores nas costas, comer em excesso, cansaço, e 85,4\% da população apresentou melhora dos sintomas após 0 tratamento. Destes 41 profissionais, em 31 mulheres, os sintomas que apresentaram melhores resultados após oito sessões de auriculoterapia foram: angústia (80\%), dores nas costas (77.70\%), exaustão física (76.90\%) e esquecimento (70\%). Nos 10 homens, referiram-se como principais sintomas: o desgaste, comer demais e dores nas costas. Após o tratamento, para "desgaste" e "comer demais" houve $80 \%$ de melhora( ${ }^{(8)}$.

No atual estudo, entre os 21 sintomas que obtiveram diferenças estatísticas, aqueles que obtiveram melhores resultados após oito sessões para agulhas semipermanentes foram: tenho taquicardia $(p=0.004)$, minha pressão arterial se altera $(p=0.001)$, sinto raiva $(p=0.001)$, sinto insegurança $(p=0.005)$ e tenho pesadelos $(p=0.005)$. Dos oito sintomas que se modificaram após o tratamento com sementes, os principais foram: meus músculos estão sempre tensos $(p=0.005)$, tenho a sensação de que vou desmaiar 
$(p=0.015)$ e sinto os olhos lacrimejantes e a visão embaçada $(p=0.013)$.

Embora a auriculoterapia com agulhas tenha produzido melhores resultados, a vantagem da auriculoterapia com sementes é que pode ser indicada naquelas condições em que o paciente não apresenta tolerância para estímulos invasivos ou em tratamento de crianças. Já se mostrou eficaz para raquialgia crônica em idosos $^{(21)}$. Mas os efeitos podem ser discutíveis ao compará-lo com outros materiais, pois foi utilizado em grupo controle como placebo, para testar a eficácia de bolinhas magnéticas em grupo experimental para idosos que sofriam de dor lombar, com resultados significativos para o grupo de intervenção quando comparados ao controle ${ }^{(22)}$.

Um conjunto de sintomas físicos e psicológicos compõe o estado de estresse vivenciado no cotidiano das pessoas. O estresse foi definido como um conjunto de reações fisiológicas que o organismo desenvolve frente a uma situação de esforço, desencadeado por estímulo ameaçador à homeostase, gerando sintomas como falta de apetite, pressão alta, desânimo e fadiga ${ }^{(23)}$. Estes sinais e sintomas encontram paralelos na MTC quanto aos diversos padrões de desequilíbrio energético de órgãos (Zheng) implicados no estresse. A acupuntura clássica está estruturada sobre bases filosóficas da medicina antiga, partindo de conceitos como Qi, Yin, Yang, Cinco Elementos, Fisiologia energética dos órgãos e vísceras (Zang Fu). Princípios energéticos, como deficiência e excesso, interior e exterior, calor e frio são conceitos sobre os quais se estrutura a realização dos diagnósticos. Tais avaliações são realizadas a partir de sinais observáveis e sintomas relatados, pela palpação do pulso radial, análise da língua (formato, cor, movimento, aspecto do revestimento) e em grande parte pela sensibilidade, experiência e conhecimento do praticante sobre os desequilíbrios entre os meridianos e sistemas ${ }^{(9)}$. Desta forma, a análise comparativa dos sintomas de estresse e diagnósticos de MTC proposto no presente estudo é um levantamento dos possíveis padrões de desequilíbrio energético, que precisariam ser, a rigor, individualizados e melhor avaliados durante o atendimento.

Os diagnósticos de MTC encontrados envolveram principalmente a desarmonia dos meridianos dos rins, coração, estômago, baço-pâncreas, fígado e meridianos tendino-musculares. Resultados similares foram encontrados em estudo realizado sobre principais diagnósticos de MTC para o Estresse Pós-traumático a partir dos sintomas descritos pelo Manual Diagnóstico e Estatístico de Transtornos Mentais (DSM-IV) e avaliados por 20 acupunturistas experientes, e testados posteriormente em 21 indivíduos com esta enfermidade ${ }^{(13)}$.

Os três pontos do protocolo de auriculoterapia não foram exatamente os pontos auriculares dos meridianos, com exceção dos rins. Para a MTC, aos rins se atribuem não somente as funções de secreção, excreção e absorção dos rins da fisiologia ocidental, mas sua influência sobre as gônadas, a suprarrenal, a produção de medula óssea e características constitucionais hereditárias. Tal relevância é atribuída a este meridiano, que é considerado fonte e sede de todo Yin e Yang que regulariza e mantém o equilíbrio energético do corpo, sendo responsável pelo nascimento, crescimento, reprodução e morte(24). Justifica-se, portanto, o uso do ponto auricular dos rins como um ponto mobilizador de recursos energéticos e de reequilíbrio durante o processo vivenciado no estresse. Os sintomas referentes aos rins estão relacionados na Tabela 4, com características importantes de deficiência e depleção energética, caracterizados pelo desânimo, cansaço, falta de forças, insegurança e disfunções sexuais.

Não foi utilizado, em especial, nenhum ponto para tratar o meridiano do fígado e vesícula biliar, responsáveis por sintomas como irritabilidade, nervosismo, dores musculares, lacrimejamento, visão embaçada e enxaquecas, quando em desequilíbrio. São muitas as funções energéticas do meridiano do fígado: circular e governar o livre fluxo de Qi por todo o corpo, governar a visão, músculos e tendões, armazenar o sangue, entre outras $^{(9)}$. Pode-se traçar um paralelo entre a função de controle e organização da circulação geral de Qi da MTC com o hipotálamo da Medicina Ocidental, que tem um papel central em orquestrar uma resposta humoral, visceromotora e somaticomotora apropriada, exercendo influência sobre as glândulas endócrinas, suprarrenais (relativo aos rins da MTC), pâncreas, tireoide entre outros $^{(12)}$. E, embora não tenha sido utilizado o ponto auricular do fígado, estômago e baço-pâncreas, o ponto Tronco Cerebral foi utilizado como um harmonizador das funções do Sistema Nervoso Central, relativo ao estresse e suas consequências sobre o sistema digestivo e cardíaco trazendo resultados positivos para sintomas como: sinto náuseas, tenho um nó no estômago, meu apetite oscila muito, tenho taquicardia, tenho a respiração ofegante.

O efeito terapêutico dos estímulos físicos e mecânicos produzidos sobre a aurícula atua sobre a inervação periférica por três principais nervos (nervo auriculotemporal, o ramo auricular do $X$ par de nervos com fibras do VII e IX par de nervos); o plexo cervical e a 
possível interferência neural central de diferentes fibras sensoriais originárias do tronco cerebral e tálamo ${ }^{(17)}$. Os pontos Tronco Cerebral, Tálamo e Hipotálamo estão localizados na mesma região da aurícula, no bordo superior da fossa intertrágica e internamente inervados por ramos dos nervos vago, facial e glossofaríngeo. 0 ponto Tronco Cerebral tem indicação para controle da atividade cardíaca, cefaleia, vômitos, náusea, respiração e temperatura corporal(12). É um ponto sedante, calmante para a mente ${ }^{(8)}$.

Quanto ao coração(Xin) da MTC, este é um órgão controlador da circulação sanguínea (bomba cardíaca) e das atividades mentais e emocionais. O Shen (mente) é a consciência organizadora, que se expressa no conjunto das funções do organismo, permitindo ao indivíduo comunicar-se e se adaptar permanentemente ao que the cerca. O psiquismo, a coerência de personalidade e aspectos mais elevados da inteligência são comandados pelo Shen ou mente. Uma condição energética de deficiência desse sistema provoca um estado depressivo, de timidez, de insegurança e incapacidade de ter uma percepção justa das situações, originando uma tendência a queixar-se sem cessar e, em casos graves, a uma desestruturação da personalidade. Embriologicamente, o coração se origina no pólo cranial do embrião, de onde também se origina o encéfalo, sugerindo que há entre eles uma conexão primitiva que pode se perpetuar por toda a vida. Distúrbios do sono, ansiedade, alteração de pressão arterial e taquicardia são sintomas relacionados a desequilíbrios deste meridiano ${ }^{(25)}$. O ponto Shenmen tem relação direta com o meridiano do coração, pois é um ponto que controla a mente, tendo a função de acalmar e relaxar. Vastamente utilizado pelos chineses para

\section{REFERÊNCIAS}

1. Fernandes SMBA, Medeiros SM, Ribeiro LM. Estresse ocupacional e o mundo do trabalho atual: repercussões na vida cotidiana das enfermeiras. Rev. Eletr. Enf. [Internet]. 2008 [acesso em: 31 mar 2014];10(2):414-27. Disponível em: http://www.fen.ufg.br/revista/v10/n2/v10n2a13.htm.

2. Coelho Neto NM, Garbaccio JL. O estresse ocupacional no serviço de Enfermagem hospitalar: reconhecimento e minimização. Interseção. 2008;1(2):71-81.

3. Silva JLL, Melo ECP. Estresse e implicações para o trabalhador de Enfermagem. Informe-se [Internet]. 2006 [acesso em: 31 mar 2014];2(2):16-8. Disponível em:

http://www.uff.br/promocaodasaude/estr.trab.pdf.

4. Seleghim MR, Mombelli MA, Oliveira ML, Waidman MA, Marcon SS. Sintomas de estresse em trabalhadoras de enfermagem de uma unidade de pronto socorro. Rev Gaucha Enferm [Internet]. 2012 [acesso em: 31 mar 2014];33(3):165-73. Disponível em: http://dx.doi.org/10.1590/S1983-14472012000300022.

5. Urbanetto JS, Silva PC, Hoffmeister E, Negri BS, Costa BE, Figueiredo CE. Workplace stress in nursing workers from an emergency hospital: Job Stress Scale analysis. Rev Lat Am Enfermagem [Internet]. 2011 [acesso em: 31 mar promover analgesia, também é um ponto antiinflamatório e antialérgico(12).

\section{CONCLUSÕES}

Os pontos auriculares utilizados neste estudo conseguiram produzir um efeito positivo sobre a diminuição do estresse nos profissionais de Enfermagem, com melhores resultados para agulhas semipermanentes do que para sementes, que não apresentou diferenças estatísticas significativas na análise intergrupos. Vinte e um sintomas de estresse obtiveram diferenças significativas entre o antes e após oito sessões no grupo agulha, porém os principais sintomas foram relativos à taquicardia, pressão arterial alterada, raiva, insegurança e pesadelos. Dos oito sintomas que se modificaram antes e após o tratamento com sementes, os principais foram: músculos sempre tensos, sensação de que vai desmaiar, olhos lacrimejantes e visão embaçada. Foram identificados os principais padrões de MTC decorrentes da análise comparativa dos sintomas: Estagnação de Qi no tórax, Estagnação de Qi do fígado, Deficiência energética de rins/coração, Desarmonia do fígado e coração, Estagnação de Qi/sangue nos meridianos tendinomusculares e fígado, Deficiência de Yin do fígado, Deficiência geral de Qi/sangue. O presente ensaio buscou contribuir para a identificação de diagnósticos de MTC no controle de estresse, fornecendo dados que permitem a sistematização de novos protocolos auriculares, cobrindo uma ampla gama de possibilidades de padrões de desequilíbrio energético nos meridianos. Porém, mais estudos se fazem necessários para que tais diagnósticos sejam confirmados e possam ser extensíveis a outros profissionais.

2014];19(5):1122-31. Disponível em: http://dx.doi.org/10.1590/S0104-11692011000500009.

6. Donovan RO, Doody O, Lyons R. The effect of stress on health and its implications for nursing. Br J Nurs. 2013;22(16):969-3.

7. Kurebayashi LFS, Gnatta JR, Borges TP, Silva MJP.

Applicability of auriculotherapy in reducing stress and as a coping strategy in nursing professionals. Rev Lat Am Enfermagem [Internet]. 2012 [acesso em: 31 mar 2014];20(5):980-7. Disponível em: http://dx.doi.org/10.1590/S010411692012000500021.

8. Giaponesi ANL, Leão ER. A auriculoterapia como intervenção para redução do estresse da equipe de enfermagem em terapia intensiva. Nursing (São Paulo). 2009;(139):575-9.

9. Lu AP, Jia HW, Xiao C, Lu QP. Theory of traditional Chinese medicine and therapeutic method of diseases. World J Gastroenterol [Internet]. 2004 [acesso em: 31 mar 2014];10(13):1854-6. Disponível em: http://www.wjgnet.com/1007-9327/full/v10/i13/1854.htm. 10. Wang Y, Ma LZ, Liu P, Liao XW. Relationship between symptoms of traditional Chinese medicine and indicator of western medicine about liver cirrhosis. J Biomed Sci Eng 
[Internet]. 2008 [acesso em: 31 mar 2014];1:104-9. Disponível em: http://dx.doi.org/10.4236/jbise.2008.12017.

11. MacPherson $H$, Altman DG, Hammerschlag $R$, Youping $L$, Taixiang W, White A, et al. Revised STandards for Reporting Interventions in Clinical Trials of Acupuncture (STRICTA): extending the CONSORT statement. PLoS Med [Internet]. 2010 [acesso em: 31 mar 2014];7(6):e1000261. Disponível em: http://dx.doi.org/10.1371/journal.pmed.1000261.

12. Landgren K. Explanatory models for acupuncture. In: Landgren K. Ear Acupuncture: a practical guide. New York: Elsevier; 2008. p 37-53.

13. Hollifield M, Sinclair-Lian N, Warner TD, Hammerschlag R. Acupuncture for posttraumatic stress disorder: a randomized controlled pilot trial. J Nerv Ment Dis [Internet]. 2007 [acesso em: 31 mar 2014];195(6):504-13. Disponível em:

http://dx.doi.org/10.1097/NMD.0b013e31803044f8. 14. Prado JM, Kurebayashi LF, Silva MJ. Efficacy of auriculotherapy for the reduction of stress in nursing students: a randomized clinical trial. Rev Lat Am Enfermagem [Internet]. 2012 [acesso em: 31 mar 2014];20(4):727-35. Disponível em: http://dx.doi.org/10.1590/S0104-11692012000400013. 15. Souza EFAA, Luz MT. Análise crítica das diretrizes de pesquisa em medicina chinesa. Hist Cienc Saude Manguinhos [Internet]. 2011 [acesso em: 31 mar 2014];18(1):155-74. Disponível em: http://dx.doi.org/10.1590/S010459702011000100010.

16. Vasconcelos EG. Stress, coping ans soziale kompetenz bei kardiovaskularen ekrankugen [tese] München: Ludwig Maxximilians Universitat in Munchen; 1984.

17. Garcia E. Definição, Função e Diagnóstico dos Pontos Auriculares. In: Garcia E. Auriculoterapia. São Paulo: Roca; 1999. p. 61-116.

18. Gnatta JR, Kurebayashi LF, Silva MJP. Atypical mycobacterias associated to acupuncuture: an integrative review. Rev Lat Am Enfermagem [Internet]. 2013 [acesso em: $31 \mathrm{mar}$ 2014];21(1):450-8. Disponível em:

http://dx.doi.org/10.1590/S0104-11692013000100022. 19. Maciocia G. Canais de acupuntura: uso clínico dos canais secundários e dos oito vasos extraordinários. São Paulo: Roca; 2007.

20. Prado JM, Kurebayashi LF, Silva MJ. Auriculotherapy effectiveness in the reduction of anxiety in nursing students. Rev Esc Enferm USP [Internet]. 2012 [acesso em: 31 mar 2014];46(5):1200-6. Disponível em:

http://dx.doi.org/10.1590/S0080-62342012000500023. 21. Vas J, Aguilar I, Campos MA, Méndez C, Perea-Milla E, Modesto $M$, et al. Randomised controlled study in the primary healthcare sector to investigate the effectiveness and safety of auriculotherapy for the treatment of uncomplicated chronic rachialgia: a study protocol. BMC Complement Altern Med [Internet]. 2008 [acesso em: $31 \mathrm{mar} 2014$ ];8:36. Disponível em: http://dx.doi.org/10.1186/1472-6882-8-36.

22. Suen LK, Wong TK, Chung JW, Yip VY. Auriculotherapy on low back pain in the elderly. Complement Ther Clin Pract [Internet]. 2007 [acesso em: 31 mar 2014];13(1):63-9. Disponível em: http://dx.doi.org/10.1016/j.ctcp.2006.10.005. 23. Doria MCS, Lipp MEN, Silva DF. O uso da acupuntura na sintomatologia do stress. Psicol. cienc. prof. [Internet]. 2012 [acesso em: 31 mar 2014];32(1):34-51. Disponível em: http://dx.doi.org/10.1590/S1414-98932012000100004. 24. Maciocia G. The foundations of Chinese Medicine: a comprehensive text for acupuncturists and herbalists. New York: Elsevier;2005.

25. Sinclair-Lian N, Hollifield M, Menache M, Warner T, Viscaya J, Hammerschlag R. Developing a traditional chinese medicine diagnostic structure for post-traumatic stress disorder. J Altern Complement Med [Internet]. 2006 [acesso em: $31 \mathrm{mar}$ 2014];12(1):45-57. Disponível em: http://dx.doi.org/10.1089/acm.2006.12.45.

Artigo recebido em 17/09/12.

Aprovado para publicação em 16/09/13.

Artigo publicado em 31/03/2014. 